\title{
Photoconversion efficiency of quantum-well solar cells for the optimum doping level of a base
}

\author{
A.V. Sachenko, I.O. Sokolovskyi \\ V. Lashkaryov Institute of Semiconductor Physics, NAS of Ukraine \\ 41, prospect Nauky, 03028 Kyiv, Ukraine
}

\begin{abstract}
Analytical expressions for the maximum obtainable photoconversion efficiency of quantum-well solar cells (QWSCs) under AM0 conditions are given. The modeling of the photoconversion efficiency of QWSCs under AM1.5 conditions using the SimWindows program is fulfilled. It is shown that the photoconversion efficiency of QWSCs with the $\mathrm{A}_{3} \mathrm{~B}_{5} p-i-n$ structure is rather low because of a low photovoltage value. To improve this situation, the base region should be doped heavily enough. Light concentration makes it possible to realize high photoconversion efficiencies for $A_{3} B_{5}$ quantum-well $p-i-n$ structures with a low background level of the base region doping. Their values are comparable to the photoconversion efficiencies for solar cells (SCs) with rather high base region doping levels.
\end{abstract}

Keywords: photoconversion efficiency, quantum well, solar cell.

Manuscript received 25.12.07; accepted for publication 07.02.08; published online 31.03.08.

\section{Introduction}

The question of the availability of quantum-well solar cells (QWSCs) is highly attended last years. Many researches cover features of QWSCs, and there are the special sections on such questions at European and world solar energy conferences for last nine years. By efforts of scientists headed by Prof. K.W.J. Barnham at the Imperial College in London, the laboratory patterns of $\mathrm{A}_{3} \mathrm{~B}_{5}$ QWSCs with high efficiency comparable with the record value in conventional $\mathrm{SCs}$ have been obtained [1-3].

However, up to now, all efforts have been concerned with the analysis and the fabrication of QWSCs with $p-i-n$ structures and a low doping of the base region. There was no comparative analysis of the photoconversion efficiency for QWSCs and conventional SCs. In this work, we present the results of a theoretical analysis of the efficiency of photoconversion at the optimum doping of the base region.

The present paper consists of three sections. Analytical expressions for the maximum obtainable photoconversion efficiency of QWSCs at AM0 conditions are obtained in the first section. The maximum obtainable photoconversion efficiency of QWSCs as a function of the quantum well bandgap for GaAs, AlGaAs, and $\mathrm{Si}$ as the barrier material is calculated using these expressions. The calculations have been carried out for both concentrated and non-concentrated illuminations.

The modeling of the photoconversion efficiency of QWSCs at a non-concentrated illumination under AM1.5 conditions is fulfilled using the SimWindows program $[4,5]$ in the second section. GaAs is considered as the barrier material with InGaAs quantum wells. It is shown that the photoconversion efficiency of QWSCs can considerably exceed the conventional efficiency of SCs under two conditions: 1) the quantum well carrier lifetime exceeds the barrier carrier lifetime; 2) the doping level of the base region does not exceed $10^{17} \mathrm{~cm}^{-3}$. However, the maximum photoconversion efficiency is achieved in conventional SCs with doping levels higher than $10^{17} \mathrm{~cm}^{-3}$.

In the third section, it is shown that concentrated illumination allows one to achieve the high photoconversion efficiency in quantum well $p-i-n$ structures with the low base region doping. This efficiency is comparable with the efficiency of SCs with highly doped base region.

Then we present a detailed description of the obtained results.

\section{Model and main results}

In the calculations of the limiting efficiency of QWSCs, we consider the solar spectrum under AM0 conditions as 
the blackbody spectrum at a temperature of $5800 \mathrm{~K}$. One electron-hole pair generation by every photon in the interband absorption range was assumed. Light reflection and contact grid shadowing were neglected. We took the photocurrent collection coefficient to be 1 . The recombination in the base region consisting of the bulk recombination in barriers, recombination in quantum wells, and "surface" recombination on the barrier - quantum well interfaces was taken into consideration. Recombination velocities are $V_{r}$ and $S$, respectively. Quantum wells are assumed to be located outside the screening area. The concentration $\Delta p$ of excess electron-hole pairs in the base region is determined as

$\Delta p=J_{S C} / q V_{\text {eff, }}$

where $V_{\text {eff }}=V_{r}+S ; J_{S C}$ is the surface short-circuit current density, and $q$ is the electron charge.

The open circuit voltage

$V_{O C}=(k T / q)\left[\ln \left(\Delta p / p_{0}\right)+\ln \left(1+\Delta p / n_{0}\right)\right]$,

where $k$ is the Boltzmann constant, $T$ is the absolute temperature, and $p_{0}$ and $n_{0}$ are, respectively, the concentrations of majority and minority current carriers.

The short-circuit current density $J_{S C}$ is a function of the barrier material bandgap $E_{g b}$, quantum wells bandgap $E_{g q}$, and the effective times of recombination $\tau_{\text {eff }}$ and quantum well carrier escape $\tau_{\text {esc }}$ :

$J_{S C}=J_{S C}\left(E_{g b}\right)+$

$+\left[J_{S C}\left(E_{g q}\right)-J_{S C}\left(E_{g b}\right)\right] \frac{\tau_{\mathrm{eff}}}{\tau_{\mathrm{eff}}+\tau_{\mathrm{esc}}}$.

We have calculated the limiting photoconversion efficiency of QWSCs under AM0 conditions by using relations (1)-(3). We set $\tau_{\text {eff }}=d_{b} / V_{\text {eff }}$, where $d_{b}$ is the base region thickness, and take $\tau_{\text {esc }}=W \sqrt{2 \pi m / k T} \exp \left(E_{a} / k T\right)$ [6] ( $W$ is the quantum well width, $m$ is the effective mass of a quantum well carrier, and $E_{a} \approx\left(E_{b}-E_{g}\right) / 2$ is the activation energy).

The band structure of a QWSC is demonstrated in Fig. 1, where Fig. 1a corresponds to a low doping of the base region and the $\Delta p<n_{0}$ condition. In this case, a strong electric field acts in the base region in the maximum power takeoff mode. Fig. $1 \mathrm{~b}$ is for a high enough doping of the base region or the $\Delta p>n_{0}$ situation. In this case, there is no electric field in the base region in the maximum power takeoff mode.

When $\Delta p<n_{0}$, the open-circuit voltage $V_{O C} \approx$ $(k T / q) \ln \left(\Delta p n_{0} / n_{i}^{2}\right)$, where $n_{i}$ is the intrinsic carrier concentration of a barrier material, and $n_{i}$ logarithmically depends on the doping level of the base region. The greater is $n_{0}$, the greater is $V_{O C}$. In the case of a high excitation level when $\Delta p>n_{0}$, the open-circuit voltage $V_{O C} \approx(2 k T / q) \ln \left(\Delta p / n_{i}\right)$, and it does not depend on the

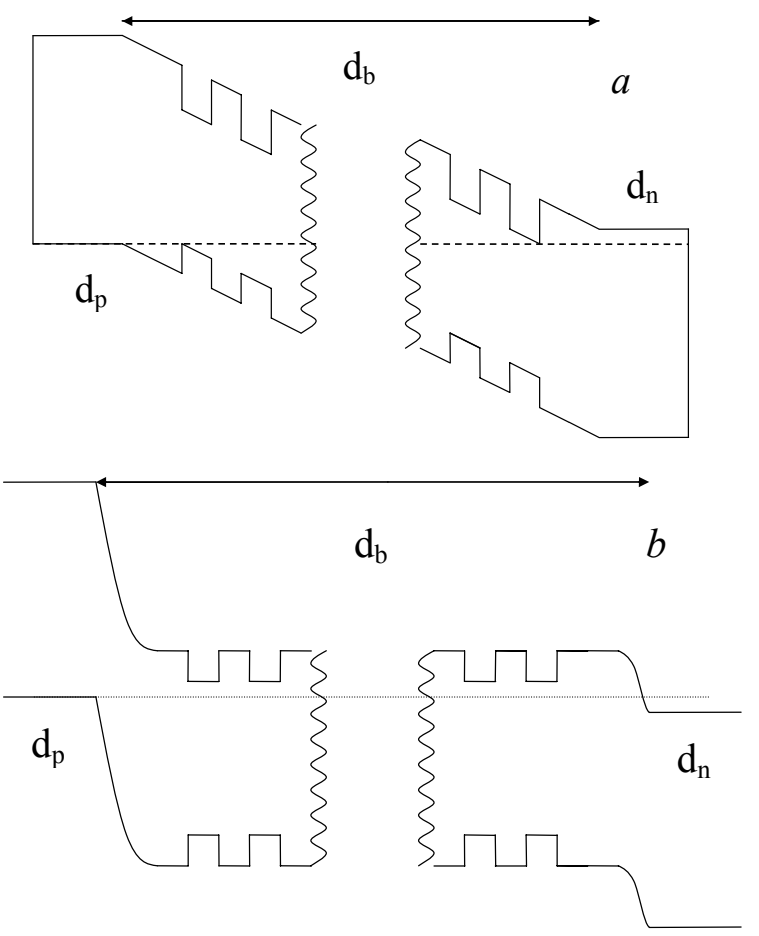

Fig. 1. Energy diagram of QWSCs: $d_{p}, d_{b}$, and $d_{n}$ are the thicknesses of the $p^{+}$, base, and $n^{+}$regions, respectively.

doping of the base region. The non-equilibrium carrier lifetime in direct band semiconductors is low enough, so the $\Delta p<<n_{0}$ criterion is satisfied under AM0 and AM1.5 conditions. High excitation levels in such semiconductors can be reached under concentrated illumination. Therefore, the QWSCs with direct band semiconductor $p-i-n$ structures and with a low doped base region have low photovoltage and photoconversion efficiency relative to those in structures with a highly doped base region. There is another situation in Si SCs, where a high excitation level can be realized under AM0 or AM1.5 condition. Thereby, the photovoltage and the photoconversion efficiency of $\mathrm{Si} p-i-n$ structures are high enough and cannot be increased by a higher doping of the base region.

In Fig. 2, we show the limiting photoconversion efficiency of QWSCs with $p-i-n$ structure versus the quantum well material bandgap $E_{g q}$ for AM0 (Fig. 2a) and at the light concentration $M=400$ ( $M$ is the degree of concentration of the illumination) (Fig. 2b). The barrier material is GaAs. Fig. 3 shows the photoconversion efficiency of SCs with $p-i-n$ structure versus the quantum well material bandgap $E_{g q}$ for AM0, the barrier material being $\mathrm{Al}_{0.35} \mathrm{Ga}_{0.65} \mathrm{As}_{5}$. The curves are built for various $V_{\text {eff. }}$.

The background doping level in Figs. 2 and 3 is $10^{15} \mathrm{~cm}^{-3}$. Efficiencies in these figures are compared with the limiting photoconversion efficiency of conventional SCs at a base region doping level of $10^{17} \mathrm{~cm}^{-3}$. 

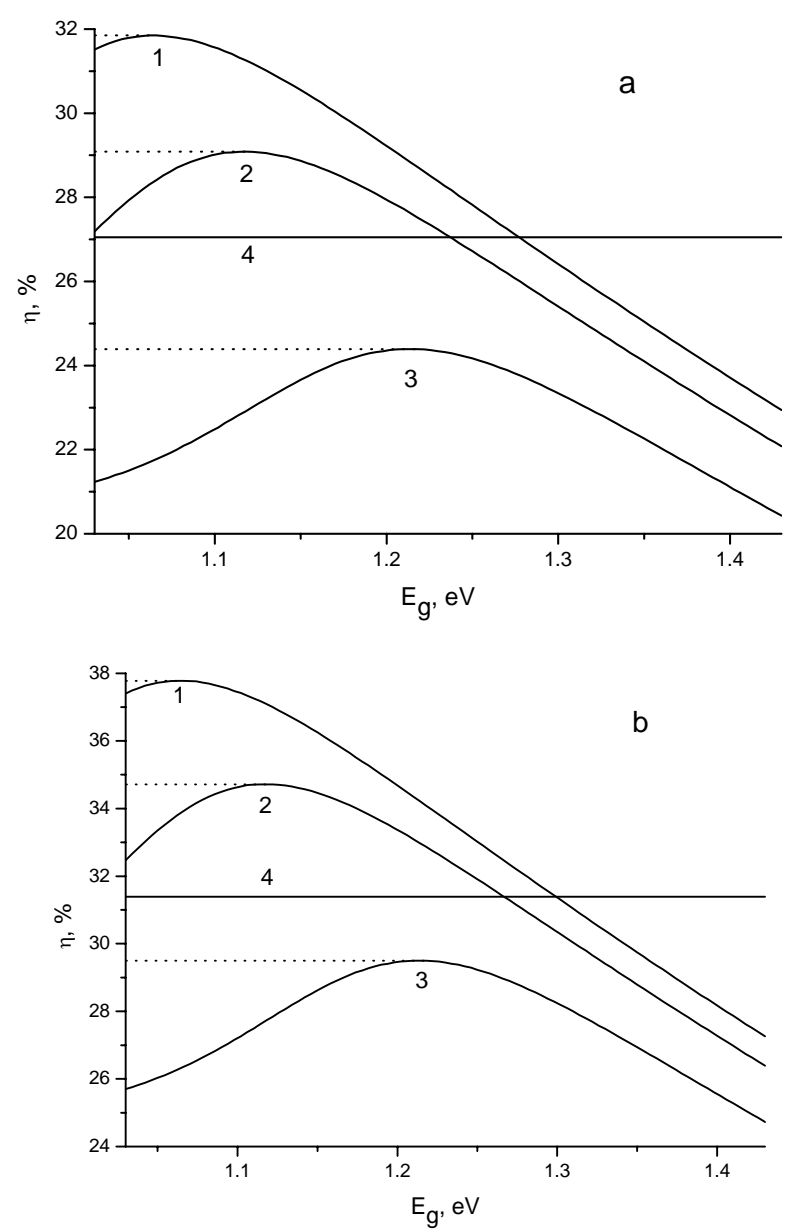

Fig. 2. Limiting photoconversion efficiency of GaAs QWSCs versus the quantum well bandgap under AM0 conditions (2a) and at the light concentration $M=400(2 \mathrm{~b}) . d_{i}=0.5 \mu \mathrm{m}, n_{0}=$ $10^{15} \mathrm{~cm}^{-3}, \quad V_{\text {eff }}=3 \cdot 10^{4} \mathrm{~cm} / \mathrm{s}(1), 10^{5}(2)$, and $10^{6}(3)$. For curve 4 (conventional SCs), $V_{\text {eff }}=10^{4} \mathrm{~cm} / \mathrm{s}, n_{0}=10^{17} \mathrm{~cm}^{-3}$.

It is seen from Figs. 2 and 3 that the limiting photoconversion efficiencies of $\mathrm{GaAs}$ and $\mathrm{Al}_{0.35} \mathrm{Ga}_{0.65} \mathrm{As}_{5}$ QWSCs have maxima depending on $E_{g q}$, by attaining 32 and $38 \%$ under concentrated AM0 conditions at $V_{\text {eff }}=$ $3 \cdot 10^{4} \mathrm{~cm} / \mathrm{s}$, so that these values exceed the limiting photoconversion efficiency of conventional SCs. However, in a more realistic case for $V_{\text {eff }} \approx 10^{5} \mathrm{~cm} / \mathrm{s}$, the photoconversion efficiency is $29 \%$ at AM0 and $35 \%$ under light concentration. These values do not differ strongly from the corresponding values for conventional SCs $(26-27 \%$ under AM0 conditions and $31 \%$ under light concentration). With the further increase in $V_{\text {eff }}$, the limiting photoconversion efficiency of QWSCs becomes less than the limiting photoconversion efficiency of conventional SCs. First of all, it covers GaAs-InGaAs and $\mathrm{Si}-\mathrm{GeSi}$ structures with high percentages of In and Ge.

We tried to take into account the fact that $V_{\text {eff }}$ increases, as $E_{g q}$ decreases. So, by taking $V_{\text {eff }}=V_{0}[1+$ $\left.a\left(\Delta E / E_{x}\right)^{m}\right]$, where $\Delta E=E_{g}-E_{g q}, V_{0}=10 \mathrm{~cm} / \mathrm{s}$, and $a$,

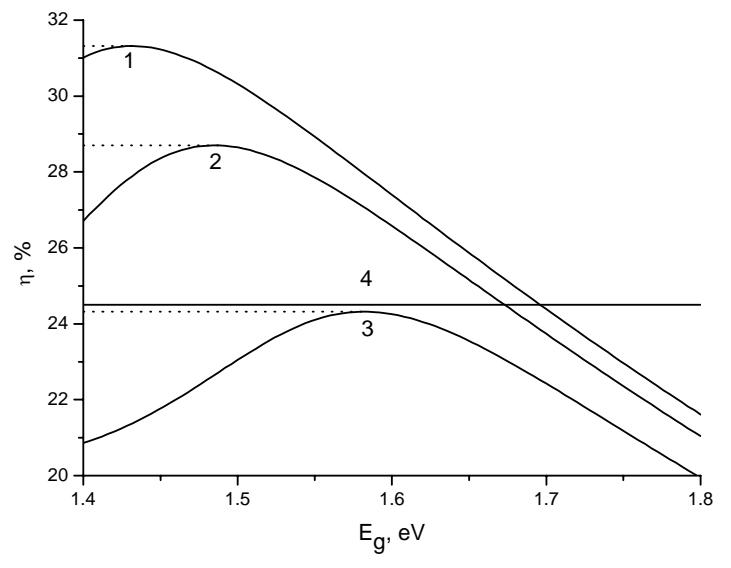

Fig. 3. Limiting photoconversion efficiency of $\mathrm{Al}_{0.35} \mathrm{Ga}_{0.65} \mathrm{As}_{5}$ QWSCs versus the quantum well bandgap under AM0 conditions. $d_{i}=0.5 \mu \mathrm{m}, n_{0}=10^{15} \mathrm{~cm}^{-3}, V_{\text {eff }}=3 \cdot 10^{4} \mathrm{~cm} / \mathrm{s}(1)$, $10^{5}(2)$, and $10^{6}(3)$. For curve 4 (conventional SCs), $V_{\text {eff }}=$ $10^{4} \mathrm{~cm} / \mathrm{s}, n_{0}=10^{17} \mathrm{~cm}^{-3}$.

$E_{x}$, and $m$ are variable parameters, we had a maximum of the limiting photoconversion efficiency of Si QWSCs versus $E_{g q}$, and this maximum was a little higher than the maximum for conventional SCs (Fig. 4). Dependences in Fig. 4 are illustrative. Generally, there are many possible choices of $V_{\text {eff }}$ as a function of $\Delta E$. The point is that these dependences illustrate the possibility of the decrease in the open circuit voltage stronger than the increase in the short circuit current with increase in $\Delta E$.

The results of numerical modeling of the photoconversion efficiency of GaAs-InGaAs QWSCs by using the SimWindows program are described in what follows. We should like to mention the unavoidable factors decreasing the photoconversion efficiency which were taken into account in the modeling. First of all, it is the bulk recombination that consists of the Shockley-Reed recombination, radiative recombination, and Auger recombination. We assumed the front and rear surface recombination velocities to be $10^{3} \mathrm{~cm} / \mathrm{s}$, what is quite a low value for direct band $\mathrm{A}_{3} \mathrm{~B}_{5}$ semiconductors. The thickness $d_{p}$ of the heavily doped $p^{+}$-region was varied and assumed to be $1 \mu \mathrm{m}$, which corresponds to the photoconversion efficiency optimum taking into account the series resistance of the $p^{+}$-region and the recombination. The thickness $d_{n}$ of the $n^{+}$-region was also $1 \mu \mathrm{m}$. The doping level of the $p^{+}$- and $n^{+}$-regions was $7 \cdot 10^{18} \mathrm{~cm}^{-3}$. Calculations were carried out for 50 InGaAs quantum wells of $9 \mathrm{~nm}$ in width separated by 6 $\mathrm{nm}$-wide barriers symmetrically disposed relative to the base region center. The base region thickness $d_{b}$ was also varied, and its mean value is assumed to be $1.15 \mu \mathrm{m}$. Losses of $12 \%$ due to reflection and contact grid shadowing were assumed.

Figure 5 illustrates the dependence of the photoconversion efficiencies of QWSCs and conventional SCs on the doping of the base region. 


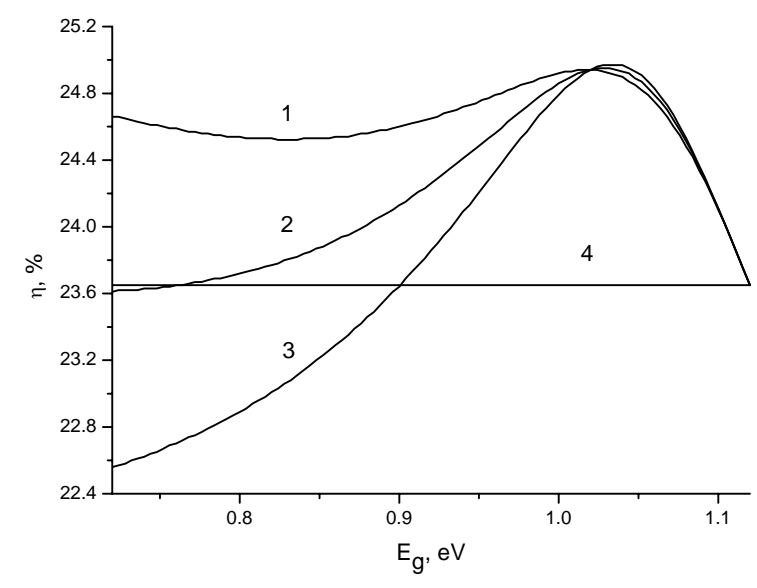

Fig. 4. Limiting photoconversion efficiency of Si QWSCs versus the quantum well bandgap under AM0 conditions. $n_{0}=10^{13} \mathrm{~cm}^{-3}, a=0.7, E_{x}=0.1 \mathrm{eV}, m=2.7(1), 3(2)$, and 3.3 (3). For curve 4 (conventional SCs), $V_{\text {eff }}=10 \mathrm{~cm} / \mathrm{s}$.

Curve 1 is plotted for conventional SCs, and curves 2 and 3 for QWSCs with $\tau_{q}$ equal to $10^{-8} \mathrm{~s}$ and $10^{-7} \mathrm{~s}$, respectively. The figure shows the significant excess of the photoconversion efficiency of QWSCs in some doping range, where the Shockley-Reed carrier lifetime for quantum wells is greater by one order of magnitude than that for the barrier material. However, the photoconversion efficiency of conventional SCs becomes greater than that of QWSCs at a doping level of (2-4) $110^{17} \mathrm{~cm}^{-3}$. The maximum photoconversion efficiency of $25.09 \%$ is practically identical to the record efficiency that has been achieved on AlGaAsGaAs structures under AM1.5 conditions (see Fig. 5, curve $m$ ).

In the modeling of QWSCs under concentrated sunlight, GaAs and InGaAs were used as materials for barriers and quantum wells, respectively. The computational parameters were identical to those in the case without light concentration.

Figure 6 shows the open-circuit voltage of QWSCs versus the light concentration $M$ for a barrier ShockleyReed carrier lifetime of $10^{-8} \mathrm{~s}$ and a corresponding quantum well lifetime of $10^{-7} \mathrm{~s}$ for different doping levels of the base region. The fraction of $\mathrm{In}$ in $\mathrm{In}_{\mathrm{x}} \mathrm{Ga}_{1-\mathrm{x}} \mathrm{As}$ was $x=0.07$. For this figure and Fig. 7, the temperature is $300 \mathrm{~K}$, which corresponds to the situation with a forced ventilation. The data were compared with those for the standard open-circuit voltage $V_{O C}=$ $(k T / q) \ln \left(J_{S C} / J_{0}\right)$, where $J_{0}$ is the saturation current density. These dependences were normalized by the selection of the value of $J_{0}$ so that the open-circuit voltages at $M=1$ coincide with those obtained as a result of the numerical modeling. Figure 6 shows on the $V_{O C}(\log q)$ scale that the numerically calculated dependences at quite low doping levels grow significantly faster than the standard linear (on this

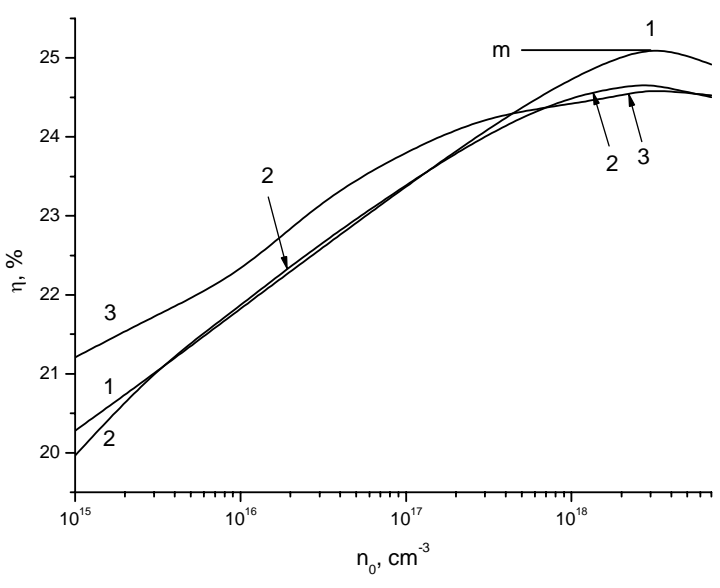

Fig. 5. Photoconversion efficiency $\eta$ versus the doping $n_{0}$ of the base region for conventional SCs (1), QWSCs with $\tau_{q}=10^{-7} \mathrm{~s}(2)$, and QWSCs with $\tau_{q}=10^{-8} \mathrm{~s}(3)$.

scale) dependences. However, the superlinearity of the dependences decreases with increase in the doping of the base region; at $n_{0}=10^{18}$, the superlinearity is not great. Finally, the open-circuit voltages for different doping levels of the base tend to approach one another with increase in the light concentration. Therefore, the opencircuit voltage of $p-i-n$ QWSCs with a low doped base region approaches that of structures with a heavily doped base region at high light concentrations. The reason consists in that the growth of a light concentration increases the concentration of excess electron-hole pairs in the $i$-region, the $\Delta p>n_{0}$ criterion becomes valid, and the decrease in the extra base- $n^{+}$-region interface band bending increases the open-circuit voltage [see Eq. (2)].

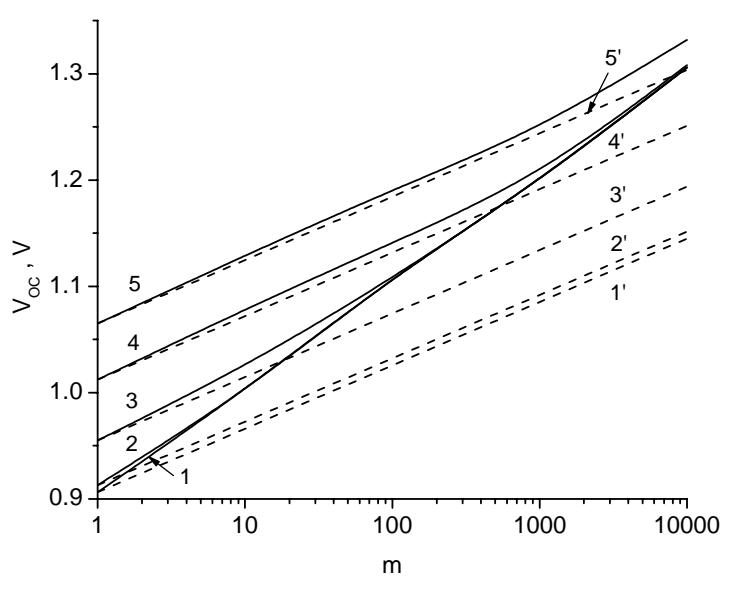

Fig. 6. Open-circuit voltage of QWSCs versus the sunlight concentration. $\tau_{b}=10^{-8} \mathrm{~s}, \quad \tau_{q}=10^{-7} \mathrm{~s}, \quad x=0.07, \quad n_{0}=$ $10^{14} \mathrm{~cm}^{-3}(1), 10^{15}(2), 10^{16}(3), 10^{17}(4), 10^{18}(5)$. Curves $1^{\prime}-5^{\prime}$ were calculated as $V_{O C}=(k T / q) \ln \left(J_{S C} / J_{0}\right)$. 


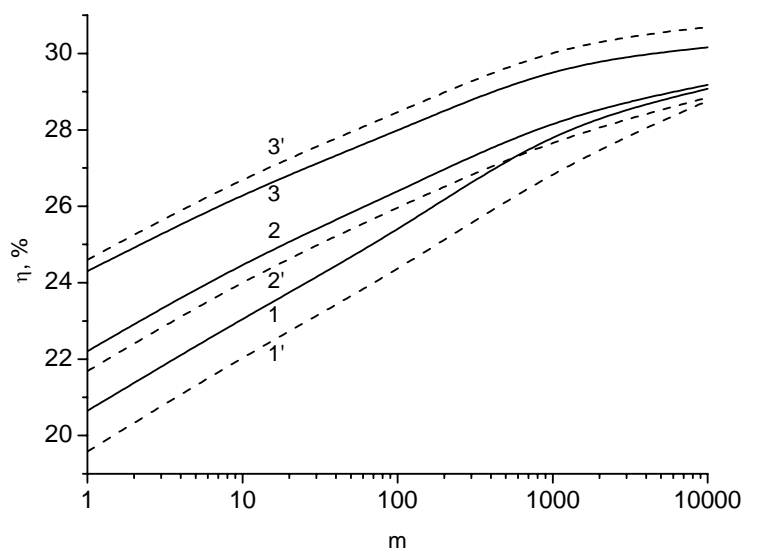

Fig. 7. Photoconversion efficiencies of QWSCs (1-3) and conventional SCs $\left(1^{\prime}-3\right)$ versus the sunlight concentration. $\tau_{b}=10^{-8} \mathrm{~s}, \tau_{q}=10^{-8} \mathrm{~s}, x=0.07, n_{0}=10^{14} \mathrm{~cm}^{-3}(1,1), 10^{16}$ $\left(2,2^{\prime}\right), 10^{18}\left(3,3^{\prime}\right)$.

The $V_{O C}(\log q)$ dependences of conventional SCs were also calculated both numerically (with the SimWindows program) and analytically. There was no qualitative difference between the open circuit voltages $V_{O C}$ of QWSCs and conventional SCs, but the values for conventional SCs were a little higher. Furthermore, the SimWindows modeling results agree with those obtained within the analytical approach. This agreement indicates both the reality of simplifications of the analytic approach and the validity of the SimWindows modeling.

Figure 7 compares the photoconversion efficiencies of QWSCs and conventional SCs in a wide range of light concentrations. It is seen that the efficiency of QWSCs is higher than that of conventional SCs up to the highest light concentration for a low doping level of the base region. At a high $\left(10^{18} \mathrm{~cm}^{-3}\right)$ doping level of the base region, the efficiency of QWSCs is lower than that of conventional SCs, but the maximum difference is only about $0.5 \%$.

\section{Conclusions}

1. The photoconversion efficiency of $\mathrm{A}_{3} \mathrm{~B}_{5} p-i-n$ QWSCs is quite low because of a low photovoltage. The base region should be doped strongly enough to increase this efficiency. However, the photoconversion efficiency of QWSCs at a high doping $\left(>3 \cdot 10^{17} \mathrm{~cm}^{-3}\right)$ of the base region is proved to be less than that of conventional SCs.

2. Light concentration enables one to get a considerable increase in the photoconversion efficiency of $\mathrm{A}_{3} \mathrm{~B}_{5} p-i-n$ QWSCs, by making it comparable to that of SCs with the highly doped base region.

3. Most effective QWSCs have a constant-sign electric field localized in narrow areas near the boundaries of the base region. There is no strong electric field in the area of quantum wells, and this situation is shown in Fig. $1 b$.

4. The difference in the photoconversion efficiencies of QWSCs and conventional $\mathrm{A}_{3} \mathrm{~B}_{5} \mathrm{SCs}$ at the same doping of the base region is small and does not exceed $5 \%$ for parameters used in this computation (see Fig. 5).

\section{References}

1. J.P. Connolly, I.M. Ballard, K.W.J. Barnham, et al., Efficiency limits of quantum well solar cells // Proc. 19th European Photovoltaic Solar Energy Conference (Paris, France, 2004), p. 355.

2. T.N.D. Tibbits, I.M. Ballard, K.W.J. Barnham, et al., Strain-balanced multi-quantum well solar cells tandem structures - first experimental results // Proc. 19th European Photovoltaic Solar Energy Conference (Paris, France, 2004), p. 3715.

3. M.C. Lynch, I.M. Ballard, A. Bessière, M. Hoyes, D.C. Johnson, P.N. Stavrinou1, T.N.D. Tibbits, I. Tongue, K.W.J. Barnham, et al., Strain balanced quantum well solar cells for high concentration applications // Proc. 20th European Photovoltaic Solar Energy Conference (Barcelona, Spain, 2005), p. 523.

4. D.W. Winston, Physical simulation of optoelectronic semiconductor devices // Ph. D. Dissertation, University of Colorado, 1996.

5. D.W. Winston //www-os.colorado.edu/SimWindows/ simwin.html.

6. M.A. Green, K. Emery, D.L. King, S. Igary, W. Warta // Progr. Photovolt.: Res. Appl. 10, p. 355 (2002).

7. A. Alemu, L. Williams, L. Bhusal, A. Freundlich, Limitations of multi-quantum well solar cells for concentrator application // Proc. 19th European Photovoltaic Solar Energy Conference (Paris, France, 2004), p. 359. 